\title{
LA GEOLOGÍA EN MÉXICO EN EL SIGLO XIX: ENTRE LAS APLICACIONES PRÁCTICAS Y LA INVESTIGACIÓN BÁSICA
}

\section{THE GEOLOGY IN MEXICO AT THE CENTURY XXI: BETWEEN PRACTICAL APLICATIONS AND BASIC RESEARCH}

\author{
Luz F. Azuela \\ Instituto de Geografía, Universidad Nacional Autónoma de México. Circuito \\ exterior s/n, Ciudad Universitaria. Delegación Coyoacán, 04510, México, D. F. \\ lazuela@igg.unam.mx
}

(Recibido: 20/11/2008; aceptado: 16/06/2009)

\begin{abstract}
This text is a journey to understand the emergency and legitimacy process of Mexican geology in the XIX century. It examines a diversity of actors intervening in that process, from the ancestors of the occupational estate of Mining; including European travelers and entrepreneurs; military technicians -during wars and foreign occupancy-; and professional scientists on the Natural Sciences in nineteenth century Mexico.

Keywords: Geology in Mexico, School of Mines, Andres Manuel del Rio, Antonio del Castillo, Geological Institute of Mexico.
\end{abstract}

RESUMEN: Este texto es un viaje para comprender la legitimación de la geología en el siglo XIX mexicano. Se examina una diversidad de actores que intervienen en el proceso, desde los antecesores en el estamento ocupacional de las minas; incluyendo viajeros y empresarios europeos; técnicos militares -durante las guerras y la ocupación extranjera-; y los científicos profesionales de las ciencias naturales del siglo XIX mexicano.

Palabras clave: Geología en México, Seminario de Minería, Andrés Manuel del Río, Antonio del Castillo, Instituto Geológico de México. 


\section{INTRODUCCIÓN}

La emergencia de la geología como disciplina independiente se abrió paso en el último cuarto del siglo XVIII y culminó en el primero del siglo XIX, luego de un arduo proceso, caracterizado por intensos debates entre legos y científicos. Este proceso se caracterizó tanto por las discusiones teóricas sobre la historia de la Tierra, como por el tránsito de la geología de una práctica profesional de interés eminentemente práctico a la legitimación de su estudio científico per se.

En las siguientes páginas abordaré el desempeño de la geología en México, desde que se empezó a impartir en el Seminario de Minería -1795como una asignatura de apoyo para formar peritos mineros hasta que alcanzó su legitimidad como disciplina independiente en el Instituto Geológico en 1891. Cuando esto ocurrió, quedaron atrás los años en que su estudio sólo se justificaba en función de sus aplicaciones prácticas y se había abierto paso a la investigación geológica como un fin en sí mismo.

\section{DE LA CÁTEDRA DE ORICTOGNOSIAA LOS VIAJEROS HUMBOLDTIANOS}

Los historiadores de la geología han definido el período comprendido entre 1780 y 1840, como la edad heroica de la geología. Tal definición comprende tres etapas diferenciadas, que incluyen los debates entre neptunistas y plutonistas -1790 a 1810-; la conformación de las primeras sociedades geológicas dedicadas exclusivamente a la exploración territorial, el registro de datos y la construcción de cartas geológicas -1807-1830-, la primera fecha corresponde a la fundación de la Geological Society of London, primera sociedad científica de la especialidad; y por último los debates entre catastrofistas -Cuvier y Buckland- y uniformistas -Charles Lyell-, que se resuelven con el triunfo del último.

La adopción de la teoría geológica de Lyell significó el reconocimiento por parte de los geólogos de que el presente es la llave para comprender el pasado. A partir de este momento se establecen las bases de un programa para el desarrollo de la nueva disciplina: la observación detallada de los procesos geológicos actuales y su explicación en términos de las teorías científicas aceptadas -física y química-.

Como se puede observar en este esquema, la caracterización de la edad heroica de la geología no se limita a registrar los adelantos teóricos de la nueva disciplina sino que alude a la constitución de una comunidad científica que discute los avances teóricos, así como la integración de los datos de los exploradores -con frecuencia mineros, agrimensores, ingenieros y militares-. De ahí, que la historia de la geología se diferencie desde sus orígenes de las historias de otras disciplinas, que pueden escribirse sin referencia alguna a sus vinculaciones con la vida práctica -v.g. la historia de las matemáticas-.

Para el análisis de la edad heroica de la geología mexicana, por lo tanto, aludiré a la geología como disciplina científica y como práctica profesional. De manera que su historia, es la historia de los individuos que efectuaron estudios geológicos; la historia de las instituciones donde se enseñó geología; la historia de los textos, estudios, colecciones y cartas; y finalmente la historia de las instituciones especializadas en la investigación que se fundaron desde el siglo XIX.

Con esta advertencia, es claro que la historia de la geología mexicana tendrá diferencias considerables con la de la geología europea. De hecho, la edad heroica de la geología mexicana abarca un período mucho mayor -1795 a 1895 - y se caracteriza no sólo por las contribuciones mexicanas a las sucesivas teorías que se formularon del otro lado del mar, sino por el empeño de nuestros geólogos en mantener las metas de la investigación científica y promover el desarrollo profesional de su disciplina en medio del fragor de la discordia política que dominó las dos terceras partes del siglo XIX.

La historia comienza entre los muros del Real Seminario de Minería fundado en 1792, en cuyo plan de estudios se incluían las Cátedras de Orictognosia, término propuesto por Werner en 1774 para referirse a la mineralogía determinativa, utilizada para la identificación de los minerales que no incluía aspectos teóricos, y Geognosia que estudiaba la estructura y composición de las rocas que forman la Tierra y podría acotarse dentro del campo de la Petrología. Ambas las impartió Don 
Andrés Manuel del Río a partir de 1795. Con estas cátedras se enseñó por primera vez la geología y dio inicio la preparación formal de los primeros cuadros técnicos y profesionales en ciencias de la Tierra en nuestro país (Rubinóvich, 1992, p. 16).

Como no contaba con textos en español para apoyar su cátedra, del Río fue dictándola con sus apuntes, que más tarde se editaron para formar la primera parte de los Elementos de Orictognosia, texto que constituye la primera obra de mineralogía producida en nuestra tierra. Valga mencionar que el texto difundía la teoría werneriana que del Río había aprendido directamente del autor, de quien había sido discípulo en la Escuela de Minas de Freiberg, en donde fue compañero de banca de Saussure, Dolomieu y Humboldt, entre otros ilustres geólogos de la gesta europea.

Con las aportaciones de los profesores y egresados del Colegio de Minería se empezaron a realizar los primeros estudios geológicos del territorio novohispano de acuerdo con los cánones europeos y se reinterpretaron los estudios previos que se habían efectuado con anterioridad. Porque como se puede suponer, tratándose de un país eminentemente minero, para el siglo XVIII, la Nueva España contaba con un buen cúmulo de datos, relaciones, estudios y cartas, realizadas por científicos, empresarios y exploradores.

Otra fuente de estudios geológicos fue la Cuenca de México, en virtud de los riesgos que implicaba la ubicación de la capital de la Nueva España en una zona lacustre naturalmente propensa a las inundaciones. De hecho, el desagüe representó un reto científico-técnico de tal magnitud, que su solución propició la publicación de un cúmulo de investigaciones sólo comparable en número al de la metalurgia. Como resultado temprano de estas obras destaca el reconocimiento de la naturaleza endorreica de la cuenca del Valle de México -1607-, que hizo Enrico Martínez, durante el diseño de un túnel para drenar las aguas de las inundaciones del río Tula, obra conocida como El tajo de Nochistongo que fue el primer proyecto geotécnico o de geología aplicada a obras de ingeniería civil desarrollado en México.

Por otra parte, la abundancia de volcanes en la Nueva España, la colosal altura de sus cimas, la configuración de sus cordilleras, la alta sismicidad de gran parte del territorio y las peculiaridades de sus suelos y de sus aguas, fueron objeto de la curiosidad y la aplicación de los europeos. De manera que desde los primeros textos de los viajeros, aparecieron descripciones detalladas de las novedades americanas e intentos de explicar sus singularidades a partir de los esquemas occidentales.

Los trabajos científicos de los viajeros llegaron a un punto culminante con la obra del barón von Humboldt, cuya expedición al continente americano -1799-1804-, incluyó una larga estancia en la capital de la Nueva España.

Como es bien sabido, después de sus experiencias en el cono sur, Alexander von Humboldt llegó al puerto de Acapulco el 23 de marzo de 1803. Se estableció en la Ciudad de México, en donde como menciona Moncada "el virrey Iturrigaray lo recibió y le abrió las puertas de oficinas y archivos, cerradas no sólo a otros extranjeros sino aún a los científicos novohispanos y peninsulares, y le facilitó un pasaporte que le permitió viajar por el reino sin ningún inconveniente" (Saladino \& Zea, 2000 , p. 34). Sus viejos compañeros de estudios de Frieberg, Andrés Manuel del Río, catedrático de Minería, y el propio Director del Seminario, Fausto de Elhúyar, pusieron a su disposición las instalaciones del Colegio de Minería para que realizara sus estudios.

Humboldt contó así con extraordinarias facilidades: información oficial privilegiada y colaboradores del más alto nivel, que le acompañaron en sus expediciones y le proporcionaron estudios, datos, colecciones naturalistas y mapas, con los que integró su Ensayo Político sobre el reino de la Nueva España -1807-1811- y su Viaje a las Regiones Equinocciales del Nuevo Continente 1807-1834-.

En ambas obras aparecen las coordenadas geográficas de los lugares que visitó; se registran observaciones astronómicas y meteorológicas; se presentan mapas y secciones de los sistemas montañosos que recorrió, con información geológica inédita; y se hacen descripciones de fauna y flora, correlacionadas con el espacio geográfico en el que se ubican. El Ensayo Político incluye además, un estudio detallado sobre las minas mexicanas, apoyado en abundantes datos estadísticos y pormenores sobre la explotación minera. 
En relación con las ciencias de la Tierra, el viaje americano le proporcionó datos sobre los componentes del campo magnético terrestre, que posteriormente elaboraría; descubrió la corriente marina en la costa occidental de Sudamérica -llamada originalmente de Humboldt y hoy conocida como corriente del Perú-; fue pionero en los estudios que relacionan las regiones geográficas con la flora y fauna locales e hizo importantes contribuciones al desarrollo de la geología a partir de sus estudios sobre los temblores de tierra y los volcanes americanos, que aparecieron publicados en sus "Volcanes y cordilleras de Quito y México".

Respecto al último punto, fue invaluable su experiencia en México en donde presenció la actividad del volcán Jorullo, de donde derivó sus conclusiones sobre el papel desempeñado por las fuerzas eruptivas en la historia y desarrollo de la corteza terrestre, que se consideraron decisivas para descartar definitivamente la hipótesis de los neptunistas.

En relación con la enseñanza de la geología, destaca su "Pasigrafía o Ensayo Geognóstico sobre el yacimiento de las rocas en los dos hemisferios”, que incluyó Andrés Manuel del Río en su Tratado de Orictognosia.

En lo que toca a la mineralogía, Humboldt dedicó todo un tomo de su Ensayo Político al estudio de los minerales económicos de México, tanto en lo que concierne a descripción física como en cuanto a los métodos de prospección, explotación, beneficio y amalgamación, así como a la organización del trabajo, la tecnología y los rendimientos de cada una de las minas.

Además del valor intrínseco de sus estudios, las obras de Humboldt promovieron el interés de científicos, exploradores e inversionistas, que viajaron a México y realizaron estudios de varias disciplinas científicas. En particular, los primeros años de vida independiente vieron desembarcar viajeros europeos vinculados con compañías mineras que pretendían establecerse en nuestro país. Durante el período 1824-1850 los principales inversionistas fueron los ingleses, seguidos por los norteamericanos y los alemanes.

La explotación minera en la variada y poco conocida topografía mexicana, exigía la atención de técnicos y científicos que efectuaran estudios geológicos y mineralógicos, que financiaron los propios empresarios. Como resultado, surgió una clase de profesionales que serían justamente los antecesores de los ingenieros geólogos, que podían hacer eficaces evaluaciones de la geología de las localidades y de la potencialidad económica de los yacimientos.

Una vez en México, los empresarios y exploradores enfrentaron las peculiaridades naturales y sociales del país, que les prodigaron materiales para escribir artículos y libros sobre sus experiencias, que acompañaron de registros y descripciones naturalistas, así como de mapas regionales, que con el tiempo conformaron un cúmulo significativo de datos científicos sobre la naturaleza y la constitución geológica de México.

La obra de mayor trascendencia científica sobre el tema que nos ocupa, fue escrita por el mineralogista Joseph Burkart (1798-1874) como resultado de una década de investigaciones en el país -que efectuó mientras dirigía la mina de Tlalpujahua-. Se trata del libro Estancia y viajes en México en los años 1825 hasta 1834, observaciones sobre el país, sus productos, la vida y costumbres de sus habitantes, así como observaciones en las ramas de mineralogía, geognosia, ciencia de minas, meteorología, geografía, obra que se publicó en alemán en dos volúmenes en el año de 1836

En la obra de Burkart aparecen estudios sobre "las ciudades y los diferentes climas, los volcanes, las fuentes termales, los distritos mineros y los edificios precolombinos" (Ramírez, 1875, p. 202) que se acompañan de tablas, mapas, cortes, mediciones, observaciones y estadísticas, que corrigieron y completaron la visión de Humboldt sobre la geografía y la naturaleza mexicanas. En la parte geológica completa sus observaciones con discusiones teóricas relacionadas con los caracteres mineralógicos y las condiciones estratigráficas de las rocas principales.

\section{LA GEOLOGÍA FLORECE EN EL FRAGOR DE LAS GUERRAS}

Mientras los peritos y viajeros ultramarinos exploraban nuestro país, el gobierno mexicano 
puso en marcha varios proyectos que involucraron estudios geográficos, geológicos y mineralógicos. Destacan los trabajos del Istmo de Tehuantepec -1823-1826-, de los alrededores de la Ciudad de México -1825- y de la frontera noreste de México y los Estados Unidos -1827-1831-, que tuvieron por objeto "observar los rasgos naturales [de cada región]; obtener información geográfica y apuntar datos exactos para los mapas" (Mendoza, 2000, p. 92). De las tres, la del Istmo de Tehuantepec se convertiría con el paso de los años, en una de las regiones mejor estudiadas del país, por su presumible potencial para la comunicación interoceánica. De la misma manera que el problema del desagüe en la Ciudad de México dio lugar al énfasis en el estudio de la Cuenca.

Entretanto, algunos gobiernos del interior efectuaron estudios regionales sobre la configuración del territorio y sus recursos naturales. Pero cada vez se hacía más evidente la necesidad de contar con investigaciones de carácter general que sirvieran como fundamento para la planeación a largo plazo y la organización de la República. Así surgió el proyecto de fundar una institución con los objetivos de construir la Carta de la República y levantar la Estadística Nacional y en 1833 se creó la Sociedad Mexicana de Geografía y Estadística $-S M G E$-, en donde se agrupó la comunidad científica e intelectual del país. De ahí que desde los primeros números de su Boletín de esta sociedad aparecieran artículos relacionados con el estudio de los diferentes tópicos que iban constituyendo el saber geológico, como disciplina científica.

En lo que respecta a los colegios de la capital, la geología se enseñaba en el Colegio de Minería, en donde se promovía la ejecución de prácticas de campo; se formaban colecciones mineralógicas de manera que los alumnos adquirieran los conocimientos prácticos para su eventual ejercicio profesional. Justamente en este Colegio comenzó a figurar el ingeniero de minas Antonio del Castillo -1820-1895-, quien desempeñaría un papel crucial en la institucionalización de la investigación geológica de México.

Del Castillo ocupó la cátedra de mineralogía y geología que dejó vacante su profesor, Andrés Manuel del Río en el Colegio Nacional de Minería. Bajo su liderazgo, comenzaron a difundirse las teorías de Lyell. De acuerdo con De Cserna (1990, p. 8), “en sus enseñanzas y actividades puso mayor énfasis en la paleontología, estratigrafía y geología de campo que su predecesor del Río". Del Castillo completaba su cátedra con trabajo de campo, que sirvió para el reconocimiento del Valle de México y sus alrededores, así como para la formación de colecciones. Sin embargo eran tiempos muy difíciles y los alumnos asistían a clases sólo cuando no había peligro en los entornos del Colegio. Eran los años de la guerra con los Estados Unidos 1847-1848-, que contra todo pronóstico, supuso grandes adelantos en la geología.

Pero antes de abundar sobre esa paradoja, es preciso recordar que cuando Antonio López de Santa Anna estampó su firma en el Tratado de Paz de Guadalupe-Hidalgo el 2 de febrero de 1848, México había perdido los estados de Nuevo México y California, que sumado al de Texas, constituían más de la mitad de su territorio -En 1853 el Tratado de la Mesilla modificó parte de los límites establecidos en el anterior-.

La firma del Tratado supuso la conformación de sendas Comisiones de Límites para efectuar los trabajos de delimitación en el campo, que dieron inicio en 1849 y terminaron en 1855. Los ingenieros mexicanos iban provistos de un pobre instrumental, con frecuencia en malas condiciones (Tamayo, 2001), que contrastaba notablemente con el equipamiento científico de los estadounidenses (Tamayo, 2000, pp. 69-70). De ahí que la Comisión mexicana se limitara a realizar trabajos cartográficos y topográficos, que aparecieron en sus informes y cartas. También se apuntaron ahí algunas notas sobre la configuración del terreno, como apuntaba Salazar Ilarregui "los cursos de los ríos, los arroyos y pantanos notables, así como de la dirección general de [los] lomeríos y los cerros aislados..." (Tamayo, 2001, p. 105), un cúmulo valioso de información que permaneció inédito debido a las prioridades del Estado en aquellos años de inestabilidad y desgobierno.

En contraste, los comisionados estadounidenses acopiaran suficiente material para publicar una amplia bibliografía sobre sus observaciones físicas de la región fronteriza. Considérese que sólo en relación con Nuevo México se registran 
un total de 19 obras que contienen descripciones y estudios geológicos de la región fronteriza (Archives de la Commission Scientifique du Mexique, 1865-1867, pp. 266-275).

Para los especialistas como Zoltan de Cserna los trabajos más valiosos se concentraron en el Levantamiento Emory (1857), que incluyó el estudio geológico de una amplia región situada, al norte y al sur de la nueva línea divisoria. A su juicio, este estudio hizo "contribuciones importantes al conocimiento de la estratigrafía, la paleontología y la petrografía de las partes septentrionales de México" y constituye el primer estudio geológico realizado en el país estadounidense que representa una aportación al patrimonio científico del mundo (De Cserna, 1990, pp. 8-9).

Pero no todo fueron pérdidas. Incluso en los peores años el interés en el desarrollo científico de México se mantuvo a contracorriente. En el terreno de la práctica destaca la formación de una Comisión para levantar la Carta Geográfica y Topográfica del Valle de México -1857-, con el objetivo de alcanzar un conocimiento más completo sobre la Ciudad de México y sus alrededores. Las dificultades políticas impidieron que los trabajos alcanzaran la totalidad de los objetivos, pero se publicaron varios trabajos parciales, entre los que destaca la Memoria para la carta hidrográfica del Valle de México, que consigna algunos datos geológicos del área (Orozco y Berra, 1862, pp. 337-512).

Hubo también en estos años viajeros que realizaron investigaciones científicas en nuestro país, entre los que destaca el prolífico naturalista suizo Henri de Saussure, quien "examinó las solfataras de la Sierra de San Andrés en el Estado de Michoacán" (De Cserna, 1990, p. 10) y construyó la Carta de México representando el Valle de Anáhuac y su vertiente oriental (Orozco y Berra, 1881). Publicó sus datos y observaciones en los libros Mémoires pour servir a l'histoire naturelle du Mexique, des Antilles et des États-Unis -1858- y Coup d'oeil sur l'hydrologie du Mexique -1862-.

Otro viajero que hizo contribuciones a la geología mexicana, fue William M. Gabb, también explorador de Costa Rica, tal como lo refieren Denyer \& Soto (2003). Gabb incursionó en territorio mexicano como miembro de la California Geological Survey, en los años sesenta del siglo
XIX, publicando un trabajo sobre los recursos minerales de Baja California, que tituló "Lower California geographical and physical features" -1868-. También escribió sobre sus fósiles cretácicos y terciarios -1869-, que luego completó con los de Sonora y Chihuahua -1872-, que aparecieron por separado, en la serie de la Geological Survey, bajo los subtítulos de "Geology" y "Paleontology." Los demás se publicaron en los Proceedings of the Academy of Natural Sciences of Philadelphia. Por las fechas, podría presumirse que algunos no se conocieron en Francia antes de la Intervención (Aguilar y Santillán, 1898, p. 86), José G. Aguilera los consideró "notables por la determinación y correlación de los horizontes geológicos [ y por] la exactitud de las determinaciones paleontológicas". A su juicio, Gabb “aportó uno de los más sólidos contingentes para el adelanto de la geología mexicana". Eran tiempos muy revueltos, pero fecundos para el desarrollo científico.

De hecho, cuando el poder estuvo en manos de los liberales bajo el liderazgo del Presidente Juárez -1861-1863-, se impulsaron iniciativas cruciales para el desarrollo científico del país. Durante su gestión, el ingeniero geógrafo Francisco Díaz Covarrubias propuso la creación de un Observatorio Astronómico en la Ciudad de México y el establecimiento de una red de observatorios meteorológicos en la República, con el fin de ampliar y sistematizar las observaciones en todo el país. La puesta en marcha de los observatorios se detuvo en 1863, después de la invasión de las tropas francesas que apoyaron el ascenso de Maximiliano de Habsburgo como Emperador.

Los liberales, acaudillados por el Presidente Juárez sostuvieron el gobierno republicano y acosaron a los franceses en una guerra sin cuartel hasta derrocar el II Imperio en 1867. La ciencia, no obstante, había recibido un estímulo perdurable

\section{DE LA GEOLOGÍA FRANCESA EN EL II IMPERIO AL INSTITUTO GEOLÓGICO DE MÉXICO (1891)}

El espíritu modernizador de Maximiliano y su actitud favorable al progreso científico se manifestaron en múltiples acciones en las que colaboró 
activamente la comunidad científica local. Durante su mandato se retomaron algunos proyectos como la Comisión del Valle de México, el Observatorio Astronómico y Meteorológico (Azuela, 2004, p. 100) y el Museo Público de Historia Natural, Arqueología e Historia; y se acogieron nuevas propuestas, como la del Ing. Antonio del Castillo sobre la formación de la Carta Geológica de los distritos minerales (Ramírez, 1890, p. 450). Al mismo tiempo, Napoleón III ordenó la conformación de la Commission Scientifique du Mexique -1864-1867-, que se proponía explorar el territorio "y conquistarlo para la ciencia" (Archives de la Commission Scientifique du Mexique, 18651867, p. 14).

El decreto de conformación de la Commission establecía como prioridad los estudios "sobre la geografía; la constitución geológica y mineralógica del país; la descripción de las especies animales y vegetales; el estudio de los fenómenos atmosféricos y de la constitución médica; el de las diversas razas, sus monumentos [y] su historia". Temas que habían sido objeto de estudios locales desde el siglo XVI y que representaban un avance sustantivo para las tareas que se emprenderían, como los propios franceses reconocieron de inmediato, aunque desconfiaban de su precisión científica y su carácter "positivo" (Archives de la Commission Scientifique du Mexique, 1865-1867, p. 266).

La Commission se dividió en cuatro comités de acuerdo a la especialidad de los estudios e inscribió la geología en el de Ciencias Naturales y Médicas. Como es de imaginar, el interés en el potencial minero del país predominó entre los invasores, de modo que los estudios geológicos y mineralógicos recibieron particular atención.

Una de las primeras tareas del Comité fue la elaboración de un instructivo para la exploración y registro de los datos, que para el caso de la geología fue elaborado por Charles Saint-Claire Deville -1814-1876-, miembro de l'Academie des Sciences (1857) y director fundador del Observatoire de Montsouris que estudió las propiedades del azufre y creó una teoría sobre los volcanes, en el caso de México inició sus disposiciones indicando que su meta sería la construcción de la Carta Geológica de México. Aunque advirtió que como ésta representaba "el resumen gráfico" de la minuciosa exploración geológica del territorio, la carta [...] dependería del conocimiento suficientemente exacto de la topografía local" (Sainte-Claire, 1864, p. 37). Es decir, se trataba de un proyecto para el mediano plazo.

Entretanto, era preciso recoger "los datos generales sobre la constitución geognóstica del nuevo Imperio Mexicano, o si se quiere, ampliar y completar los que se deben a un pequeño número de observadores, entre los que habría que citar en primera línea, después de Alexandre von Humboldt, a Burkart, de Gerolt y de Berghes" (Sainte-Claire, 1864). Sainte-Claire ordenó que sólo "en los casos particulares y para las regiones que presentaran un interés capital, ya fuera desde el punto de vista de los fenómenos eruptivos, o desde el punto de vista de la geología estratigráfica o paleontológica", los expedicionarios podrían recurrir "al apoyo de los trabajos locales" (Sainte-Claire, 1864).

El geólogo francés manifestó su interés en "el estudio químico de las emanaciones volcánicas y la descripción de los restos orgánicos de los terrenos estratificados". El "Instructivo" prosigue con la enumeración de los objetos de estudio que debían atender los expedicionarios. Destacó aquí la identificación y estudio de las vetas metalíferas, "sin ninguna duda, la mayor riqueza natural de México"; de los veneros de aguas minerales y las "chimeneas volcánicas que forman los puntos culminantes del Nuevo Mundo"; en ambos casos los reportes se debían acompañar de dibujos y fotografías cuando fuera posible, demandó la formación de colecciones de minerales; y enfatizó el interés en "recoger" meteoritos, o "por lo menos muestras" para su análisis en el laboratorio. A continuación Sainte-Claire aconseja la "colecta de todos los datos existentes en el país, impresos o manuscritos [...] sobre la geografía, la topografía, la geología y la mineralogía de México" (Sainte-Claire, 1864).

Era sin lugar a dudas un ambicioso programa que los franceses no terminaron, pero alcanzaron óptimos resultados. De acuerdo con Maldonado Koerdell la geología fue el área en la que se efectuaron "los mejores y más amplios trabajos" de la CSM (Maldonado, 1965, p.172). A la productividad bibliográfica habría que añadir las colecciones y especímenes que fueron a dar a París para enriquecer los acervos de sus centros científicos. 
Tal vez el mejor indicador del volumen de datos que se acopiaron, fue el traslado del meteorito de Charcas (de $780 \mathrm{~kg}$ ) que condujo Bazaine hasta el Museo de París.

Desde luego, no todo fue miel sobre hojuelas, como puede leerse en cualquier historia de México. Pues aún sin mencionar los horrores de la guerra, en esta época se instrumentaron proyectos que pusieron nuevamente en riesgo la integridad del país: Juárez andaba vendiendo la Baja California en Washington; Bazaine quería establecer colonias francesas en Sonora y Chihuahua, en asociación con el empresario norteamericano William Gwin; y Maximiliano daba tierras a los desertores de la Guerra de Secesión (González, 1993, pp. 456-526).

Paradójicamente, el patrimonio científico se benefició nuevamente de los riesgos, ya que todas estas acciones se materializaron en un incremento significativo del reconocimiento territorial y del estudio de su contexto geológico. Además, el acopio de las obras científicas locales que retuvieron los invasores dio lugar a la incorporación de los productos de la ciencia mexicana al acervo de la ciencia occidental del siglo XIX.

En este sentido habría que reconocer que la presencia de los franceses tuvo un significado que rebasó los objetivos de la empresa imperial, ya que la interacción y colaboración continua de los científicos europeos con los mexicanos renovó los métodos de investigación; incorporó nuevos objetos de estudio; integró instrumental y bibliografía de actualidad a las instituciones; y sobre todo, mostró las ventajas de una innovadora forma de organización de la práctica científica que se mantuvo como modelo en los años subsiguientes.

Para el tema que nos ocupa, destaca la transformación del antiguo Colegio de Minería, en una moderna Escuela de Ingenieros, en la que se ofrecieron alternativas a las carreras tradicionales: Además de formar Ingenieros de Minas, la Escuela ofrecía programas para Ingenieros Mecánicos, Ingenieros Civiles, Ingenieros Topógrafos e Hidromensores e Ingenieros Geógrafos e Hidrógrafos (Ramírez, 1890, p. 490). Los resultados de la renovación se hicieron evidentes de inmediato, pues como señala Mílada
Bazant entre 1869 y 1873 hubo un incremento de casi $150 \%$ en el número de alumnos inscritos en Ingeniería (Arce et. al., 1982, p. 222). Cifra que puede interpretarse como un indicador de las transformaciones en la ciencia mexicana que se materializarían en los siguientes años.

La geología se continuó impartiendo en la carrera de Minas, cuyos alumnos debían además aprobar química aplicada, mineralogía, metalurgia y paleontología. En todos los casos la enseñanza se apoyó con prácticas de campo y laboratorio, por lo que continuaron realizándose viajes y exploraciones geológicas y mineralógicas con propósitos didácticos.

Sin embargo, el estudio sistemático del territorio continuaba pendiente, pues hasta entonces ni siquiera se contaba con una carta general de la República ni con las cartas particulares. Estas limitaciones se subsanarían en el último cuarto del siglo XIX con la creación de organismos e instituciones especializadas, que culminarían con la fundación del Instituto Geológico de México.

Como señalo en otros textos, la consolidación institucional de la geología y su profesionalización en México, se inscribe dentro del mandato del Gral. Porfirio Díaz -1876-1911-, quien estableció más de una veintena de instituciones de carácter científico, que transformaron la práctica y el pensamiento científicos de México (Rodriguez-Sala, 1996, p. 73-84). Su primera iniciativa fue la formación de un Observatorio Astronómico -1876- con el fin de apoyar la construcción de una cartografía de precisión, este proyecto se completó en 1877 con la creación de una Comisión Geográfico-Exploradora del Territorio Nacional (CGE), a cargo del Gral. Agustín Díaz, que levantaría la Carta de la República y las cartas particulares.

En relación con la investigación geológica, la CGE abarcaba el objetivo de "dar a conocer [el territorio nacional] bajo todos sus aspectos" y tenía la obligación de hacer acto de presencia en donde acontecieran "los fenómenos Físicos Accidentales que pudieran presentarse", para estudiarlos. Sin embargo, la única relación material de la CGE con los estudios geológicos fue la cartografía que completó, en tanto que herramienta básica e invaluable para el desarrollo de la investigación. 
La formación profesional de los geólogos continuó impartiéndose en la Escuela de Ingeniería, aunque con modificaciones sustantivas en los planes de estudio. En el de 1881, se reunió la mineralogía, geología y paleontología en una sola materia y continuaron con las clases de química aplicada y metalurgia, a la que se añadió teoría y práctica de labores de minas. Se incluyó la carrera de ensayadores y apartadores, quienes cursaban análisis químico, incluyendo la docimasia y mineralogía.

Sin embargo, el hecho de que la enseñanza de la geología estuviera dirigida a la formación de ingenieros de minas, limitaba las posibilidades de la investigación. Para ello se requería de una escolaridad formal, sistemática y especializada en la disciplina y la existencia de un organismo que dirigiera la investigación y promoviera la discusión de los avances que se iban alcanzando en el nivel local y foráneo.

El personaje que pugnó por alcanzar estas metas fue el Ing. Antonio del Castillo, quien después de haber representado a México en el III Congreso Internacional de Geología, que se efectuó en Berlín en 1885, presentó la propuesta de crear un Instituto Geológico con el objetivo de elaborar la Carta Geológica de México para presentarla en el siguiente congreso, en Londres en 1888.

Del Castillo sabía que la carta geológica tendría poco interés para los políticos, de modo que en su justificación procuró indicar que el instituto tendría el propósito de "practicar el estudio geológico del territorio, dando a conocer éste desde los puntos de vista científico e industrial" (Rubinóvich, 1992, p. 26). Y para garantizar la aprobación de su iniciativa, del Castillo la vinculó hábilmente con la invitación a la Exposición Universal de París de 1889, instaurándose una Comisión Geológica con el único objetivo de editar la Carta Geológica del país para presentarla en la Feria.

La prueba material del interés del gobierno por alcanzar los laureles de la modernidad científica, fue la celeridad con que se puso en marcha el esfuerzo colectivo que implicó la conclusión del Bosquejo Geológico, la Carta Minera de la República y la organización de una colección de 4,000 ejemplares de minerales, rocas y fósiles.
A éstos, se sumaron muestras de todos los meteoritos conocidos, con los datos de su exacta ubicación geográfica y también modelos de cartón de "los dos meteoritos de Chupaderos y el de Zacatecas, conjuntamente con los de Oaxaca y los que se resguardaban en el Museo Nacional" (Ruvinóvich et. al., 1998, p. 32).

Aquí fue decisivo el puesto de Antonio del Castillo como Director de Ingeniería, ya que la Escuela proporcionó el local de la Comisión y recurrió a sus estudiantes y egresados para la integración del personal nominal y voluntario, pues obviamente las prácticas se orientaron a la consecución del ambicioso proyecto. Desde luego, la dirección del proyecto recayó en el propio del Castillo, quien incluyó como geólogo auxiliar a José G. Aguilera, a quien se sumaría después Ezequiel Ordóñez -aún estudiante (Ruvinóvich et. al., 1998, p. 34).

Por parte del Ministerio de Fomento, del Castillo recibió un sólido espaldarazo: el secretario Pacheco no escatimó ni un peso y ordenó la colaboración de todos los organismos del ministerio mediante la disposición de cartas y colecciones mineralógicas. De esta manera, no sólo se integró la Carta de la República y otras cartas particulares de la CGE, sino buen número de fósiles y rocas de sus colecciones. También se incluyeron las cartas geológicas del Istmo de Tehuantepec (Barroso, 1874, 1879); los datos, especimenes y colecciones de las Comisiones de Límites de Guatemala y Norteamérica; los de las Comisiones de Sonora y Baja California, a cargo de Joaquín M. Ramos, quien publicó un "Informe...", que incluye datos sobre la geología y la importancia industrial de los placeres auríferos; así como los perfiles geológicos de las líneas tendidas por el Ferrocarril Nacional Mexicano.

Además, los miembros de la Comisión aprovecharon las facilidades de la extensa red ferroviaria, que para 1888 sumaba alrededor de 11,500 $\mathrm{km}$, para realizar expediciones en las que verificarían datos y completarían los vacíos en el patrimonio. Se tomó especial cuidado en "recoger datos relativos a las minas en trabajo (sic) y la situación de los centros mineros [...] para formar, en un esqueleto de la Carta Geográfica de la República, la Carta Minera" (Aguilera, 1896, p. 12-14). 
Ya en París, la riqueza científica reunida le valió a México la Medalla de Oro de la Exposición.

Cuando finalmente se estableció el Instituto Geológico en 1891, Antonio del Castillo tenía setenta años de edad y más de cuarenta de desempeñarse como "geólogo". Su biografía profesional había transitado de la orictognosia de Werner a la geología de Lyell y su práctica había peregrinado por la vía franca de la minería, mientras efectuaba las traducciones que le permitieron ejercer la geología.

Para su fortuna, vio la materialización de sus sueños en el Instituto y todavía alcanzó a dirigirlo durante cuatro años e incluso pudo paladear su inserción en las redes internacionales cuando llevó su representación al V Congreso Internacional de Geología en Washington en 1891. En ese lapso se dieron a la imprenta numerosos trabajos que manifestaron la importancia del nuevo organismo dentro del sistema institucional de las ciencias y el valor social y cognitivo de la geología como disciplina independiente. Y más aún, una vez que se contó con un presupuesto propio; un grupo de profesionales que devengarían un salario por efectuar estudios específicos; y un local equipado y acondicionado con estos únicos objetivos, la geología mexicana entró de lleno a una nueva era.

Para 1903 México había adquirido tal prestigio internacional que le fue concedida la sede del X Congreso Internacional de Geología que se efectuaría en 1906. El Congreso sirvió de impulso para la intensificación de los trabajos mexicanos, además de que obligó al gobierno a incrementar recursos para la investigación geológica y sobre todo para concluir las obras del edificio Instituto Geológico, ubicado enfrente de la Alameda de Santa María. Esta edificación tiene un significado muy especial dentro del proceso de institucionalización de la geología mexicana, pues se diseñó de acuerdo con las necesidades y objetivos de la investigación de la disciplina.

Pero además, el lujo y la exquisitez de su arquitectura dieron fe del lugar de la geología entre las prioridades nacionales y la importancia que había cobrado en el entramado de las disciplinas científicas y las prácticas profesionales. El edificio, de estilo renacentista, fue construido por el arquitecto Carlos Herrera López entre 1901 y
1906. En la fachada destacan elementos decorativos a base de figuras con temas paleontológicos, botánicos y zoológicos, labrados en alto y bajo relieve. En el interior sobresale la escalera, como un ejemplar único del estilo art nouveau.

Habían quedado atrás los años en que la geología era una práctica científica realizada por voluntarios y científicos amateurs o como auxiliar para estudios de otros campos disciplinarios.

\section{CONCLUSIONES}

El estudio presentado muestra que en el desarrollo y demarcación de la geología participaron iniciativas provenientes de diferentes actores sociales, en las que destacaron los hombres de ciencia, pero también militares, empresarios, funcionarios civiles y algún aventurero. También se pone de manifiesto la influencia de las relaciones internacionales en el devenir de la práctica científica y se subraya el papel de las metrópolis en la definición de los objetivos de investigación territorial.

El marcado acento utilitario de su práctica y la eventual legitimación de la geología como disciplina científica, fue producto de negociaciones e intercambios entre sus practicantes y promotores, que fueron dejando su huella en los objetivos de la investigación geológica del siglo XIX. De esta manera, la querella entre la ingeniería de minas y la investigación geológica per se, intervino en la prefiguración de los contenidos cognitivos de la geología mexicana y marcó el destino de las nuevas generaciones de especialistas en el marco institucional de la Escuela de Ingenieros.

\section{REFERENCIAS BIBLIOGRÁFICAS}

AGUILERA, J., 1896: Bosquejo geológico de México.- Tipográfica de la Secretaría de Fomento: 4-6.

AZUELA, L., 1995: La institucionalización de la meteorología en México a finales del siglo XIX.- En: RODRÍGUEZ-SALA, M. 
\& MONCADA, J. (coords.): La cultura científico-tecnológica en México: nuevos materiales interdisciplinarios.- UNAM: 99-105.

AZUELA, L., 1996: La institucionalización de las ciencias en México durante el porfiriato.- En: RODRÍGUEZ-SALA, M. (coord.): Tres etapas del desarrollo de la cultura científico-tecnológica en México.UNAM: 73-84.

AZUELA, L., 2004: Francisco Díaz Covarrubias y la Ingeniería en México.- En: RODRÍGUEZ-SALA, M. (coord.): Del estamento ocupacional a la comunidad científica: astrónomos-astrólogos e ingenieros (siglos XVII al XX).- IIS-UNAM: 243-267.

BARROSO, A., 1874: Memoria sobre la geología del Istmo de Tehuantepec.- Anales del Ministerio de Fomento, 3: 245-330.

BARROSO, A., 1879: "Memoria sobre la geología del Istmo de Tehuantepec".- En FERNÁNDEZ, L.: Informe sobre el reconocimiento del Istmo presentado al Gobierno Mexicano, 61-106.

BAZANT, M., 1982: La república restaurada y el porfiriato.- En: ARCE, G.F., BAZANT, M., STAPLES, A,.TANCK, D., \& VÁZQUEZ, J.Z.: Historia de las profesiones en México, COLMEX: 131-222.

CSERNA, Z. de, 1990: La evolución de la geología en México (1500-1929).- Rev. del Inst. de Geol., 9(1): 1-20.

CSM, 1865-1867: Archives de la Commission Scientifique du Mexique.- 467 págs. Ministère de l'Instruction Publique, 3 vols.

DENYER, P. \& SOTO, G., 2003: Contribución de William M. Gabb a la geología de
Costa Rica del siglo XIX.- En Peraldo G. (comp.): Ciencia y técnica en la Costa Rica del siglo XIX.- Editorial Tecnológica de Costa Rica, 301-322

GONZALEZ, M., 1993: Los extranjeros en México y los mexicanos en el extranjero, 1821-1970.- 600 págs. (vol. I), COLMEX, México.

MALDONADO-KOERDELL, M. 1965: La obra de la Commision Scientifique du Mexique.En: ARNAIS Y FREG, A. \& BATAILLON C. (eds.): La intervención francesa y el imperio de Maximiliano cien años después, 1862-1962, Asociación Mexicana de Historiadores.- Inst. Francés de Amér. Latina, 160-182.

MENDOZA, H., 2000: Las opciones geográficas al inicio del México independiente.En: MENDOZA, H. (coord.): México a través de los mapas. UNAM-Plaza y Valdés: 89-110.

MONCADA, J., 2000: Humboldt y el desarrollo de la cartografía mexicana.- En: ZEA, L. \& SALADINO, A. (coords.): Humboldt y América Latina.- IPGH-UNAM-FCE: 27-40.

OROZCO Y BERRA, M., 1862: Memoria para la carta hidrográfica del Valle de México.Boletín de la Sociedad Mexicana de Geografía y Estadística, 9: 337-512.

OROZCO Y BERRA, M., 1881 [facsímil publicado en 1973]: Apuntes para la historia de la geografía en México [facsímil de la edición mexicana de 1881].- 503 págs. Biblioteca Mexicana de la Fundación Miguel Alemán.

RAMÍREZ, S., 1875: Discurso en elogio fúnebre del Doctor H. José Burkart, pronunciado en la sesión que la Sociedad Mexicana de Geografía y Estadística celebro en honor de éste sabio, por el socio... el 15 de 
mayo de 1875.- En: Boletín de la Sociedad Mexicana de Geografía y Estadística, 2: 195-204.

RAMÍREZ, S., 1890: Datos para la historia del Colegio de Minería, recogidos y compilados bajo la forma de efemérides por su antiguo alumno el Ingeniero de Minas.- SEFIUNAM [Facsímil publicado en 1982].

RUBINÓVICH, R., 1992: Andrés Manuel del Río y sus Elementos de Orictongnosia de 1795-1805.- En: DEL RÍO, A.: Elementos de Orictognosia, UNAM: 3-70.
RUBINOVICH, R. \& LOZANO, M. \& MENDOZA, H., 1998: Ezequiel Ordoñez. Vida y Obra (1867-1950).- 315 págs. El Colegio Nacional. México.

TAMAYO, L., 2000: La frontera MéxicoEstados Unidos. La conformación de un espacio durante el siglo XIX.- 271 págs. FFyL-UNAM, [Tesis Ph.D.].

TAMAYO, L., 2001: La geografía, arma científica para la defensa del territorio.- 188 págs. UNAM-Plaza y Valdés. 